(C) 2012 IEEE. Personal use of this material is permitted. Permission from IEEE must be obtained for all other uses, in any current or future media, including reprinting/republishing this material for advertising or promotional purposes, creating new collective works, for resale or redistribution to servers or lists, or reuse of any copyrighted component of this work in other works. 


\title{
Channel Covariance Information Based Transceiver Design for AF MIMO Relay Systems with Direct Link
}

\author{
Lenin Gopal ${ }^{\dagger}$, Yue Rong* and Zhuquan Zang ${ }^{\dagger}$ \\ ${ }^{\dagger}$ Department of Electrical and Computer Engineering, \\ Curtin University, CDT 250, 98009, Miri, Sarawak, Malaysia \\ Email: lenin@curtin.edu.my, zqzang@curtin.edu.my \\ *Department of Electrical and Computer Engineering, \\ Curtin University, Bentley, WA 6102, Australia \\ Email: y.rong@curtin.edu.au
}

\begin{abstract}
In this paper, we propose a design scheme for amplify-and-forward multiple-input multiple-output (AF MIMO) relay system with direct link to minimize the mean-squared error (MSE) of the signal estimation at the destination. In the proposed design scheme, an optimal precoding matrix is derived with the assumption that the full channel state information (CSI) of the source-relay link and partial channel state information such as channel covariance information (CCI) of the relay-destination link are available at the relay. In practical cases, if the destination is closer to the source, the source-destination link cannot be ignored. Hence, in this paper, we assume that the relay knows the partial channel state information of the source-destination link. Based on this assumption, an iterative optimal covariance algorithm is developed to achieve the minimum MSE of the signal estimation at the destination. In order to reduce computational complexity of the proposed optimal covariance algorithm, a suboptimal covariance algorithm is proposed. A numerical example shows that the developed optimal covariance algorithm outperforms the conventional CCI based MSE algorithms.
\end{abstract}

\section{INTRODUCTION}

Recently, cooperative wireless communications attract much research interest. By deploying a wireless relay in cooperative wireless communications, wireless networks coverage area can be extended and reliable and cost effective wireless network applications can be provided. In cooperative wireless communications, a relay can be deployed inside a building or tunnel to mitigate the effects of shadowing.

Two types of relaying schemes, decode-and-forward (DF) and amplify-and-forward (AF), have been proposed in [1]-[3]. In DF strategy, the relay decodes the information received from source and forwards the re-encoded signal to the destination. Whereas in AF strategy, the relay amplifies the received signal from source and retransmits the signal to the destination. When compared with the DF strategy, the AF strategy has a lower computational complexity and is easy to implement in the cooperative environment.

A relay precoding algorithm [4] and [5] for AF based multiple-input multiple-output (AF MIMO) relay has been developed to maximize the capacity of the source-destination link. In this algorithm, a precoding matrix is multiplied with

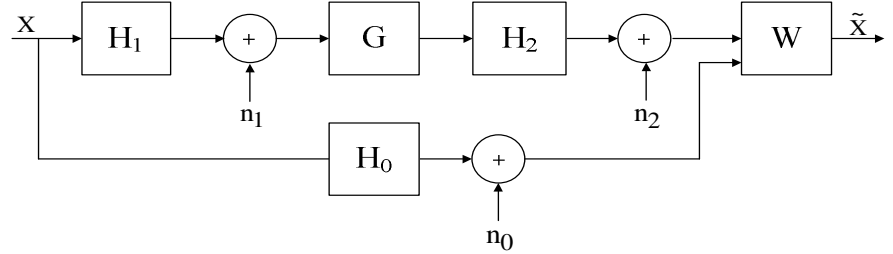

Fig. 1. AF MIMO relay system

the received signal at the relay for further signal processing. Recently [6]-[10], the precoding matrix was proposed to minimize the receiver estimation error which is known as meansquared error (MSE) of the signal at the destination node. The optimal precoding matrix design was investigated well in [9][11] for AF MIMO relay system with the assumption that the relay knows the full channel state information (CSI) of the source-relay, source-destination and relay-destination links.

In practice, the environment is mostly surrounded by scatters and shadowing effects. Due to the scattered and shadowing environments, the received signal is uncorrelated at the destination. Hence, the full CSI of the relay-destination link and the source-destination link is difficult to obtain at the relay node. For this model, the channel covariance matrix is more suitable than the instantaneous channel matrix.

In [12] and [13], optimal precoder is designed for maximizing the ergodic capacity of the AF MIMO relay system with the assumption that the channel covariance information (CCI) of the relay-destination link is available at the relay node. Recently, minimum mean-squared error (MMSE) based estimators are investigated in [14] and [15] with the assumption that the relay knows the covariance channel information of the relay-destination link. However, the optimal precoding matrix with the direct link was not investigated in [14] and [15]. In practice, the source-destination link provides valuable spatial diversity to the AF MIMO relay system and should not be ignored.

In this paper, an iterative based optimal covariance al- 
gorithm is proposed to minimize the MSE of the signal estimation at the destination in an AF MIMO relay system with direct link. Considering that the computational complexity of the developed optimal covariance algorithm may be high for practical implementation of the relay system, we propose a suboptimal covariance algorithm. In the proposed two algorithms, we assume that the relay knows the full CSI of the source-relay link and the CCI of the relay-destination link and the direct source-destination link. Simulation results verify the performance of the proposed optimal and suboptimal covariance based MSE algorithms.

The rest of the paper is organized as follows. The system model for the proposed precoding matrix design for an AF MIMO relay system is introduced in Section II. The optimal precoder design schemes are proposed in Section III. In Section IV, we present some numerical examples. Section V concludes the paper.

\section{SyStem MODEL}

Let us consider a typical three node AF MIMO relay system as shown in Fig.1, where $N_{S}, N_{R}$ and $N_{D}$ denote the number of antennas at the source, relay and destination, respectively. It is assumed that there is a direct link between the source and destination. The data transmission between the sourcedestination link is completed in two time slots. During the first time slot, the source transmits $\mathbf{x}$. The received signal at the destination and the relay during the first time slot is given by

$$
\begin{aligned}
& \mathbf{y}_{0}=\mathbf{H}_{0} \mathbf{x}+\mathbf{n}_{0} \\
& \mathbf{y}_{1}=\mathbf{H}_{1} \mathbf{x}+\mathbf{n}_{1}
\end{aligned}
$$

where $\mathbf{H}_{0} \in \mathbb{C}^{N_{D} \times N_{S}}$ is the channel matrix of the direct source-destination link, $\mathbf{x} \in \mathbb{C}^{N_{S} \times 1}$ the transmitted vector with covariance matrix $E\left\{\mathbf{x x}^{H}\right\}=\sigma_{x}^{2} \mathbf{I}_{N_{S}}, \mathbf{n}_{0} \in \mathbb{C}^{N_{D} \times 1}$ the circularly symmetric complex Gaussian noise vector with zero mean and unit variance matrix, $\mathbf{H}_{1} \in \mathbb{C}^{N_{R} \times N_{S}}$ the channel matrix of the source-relay link, $\mathbf{n}_{1} \in \mathbb{C}^{N_{R} \times 1}$ the circularly symmetric complex Gaussian noise vector with zero mean and covariance matrix $E\left\{\mathbf{n}_{1} \mathbf{n}_{1}^{H}\right\}=\sigma_{1}^{2} \mathbf{I}_{N_{R}}$. Here $E[$.] denotes the statistical expectation.

The received signal at the destination in the second time slot is given by

$$
\mathbf{y}_{2}=\mathbf{H}_{2} \mathbf{G H}_{1} \mathbf{x}+\mathbf{H}_{2} \mathbf{G n}_{1}+\mathbf{n}_{2}
$$

where $\mathbf{H}_{2} \in \mathbb{C}^{N_{D} \times N_{R}}$ is the channel matrix of the relaydestination link, $\mathbf{G} \in \mathbb{C}^{N_{R} \times N_{R}}$ a precoding matrix of the relay, $\mathbf{n}_{2} \in \mathbb{C}^{N_{D} \times 1}$ the circularly symmetric complex Gaussian noise vector with zero mean and covariance matrix $E\left\{\mathbf{n}_{2} \mathbf{n}_{2}^{H}\right\}=\sigma_{2}^{2} \mathbf{I}_{N_{D}}$. In a more compact way, the signal models (1) and (2) for the AF MIMO relay system can be written as

$$
\begin{aligned}
\mathbf{y} & \triangleq\left[\begin{array}{l}
\mathbf{y}_{2} \\
\mathbf{y}_{0}
\end{array}\right] \\
& =\left[\begin{array}{c}
\mathbf{H}_{2} \mathbf{G H}_{1} \\
\mathbf{H}_{0}
\end{array}\right] \mathbf{x}+\left[\begin{array}{c}
\mathbf{H}_{2} \mathbf{G n}_{1}+\mathbf{n}_{2} \\
\mathbf{n}_{0}
\end{array}\right] .
\end{aligned}
$$

We assume that the relay knows the full CSI of the sourcerelay link and CCI of the relay-destination link and the direct source-destination link. However, the channel information is unavailable to the source. Let us introduce

$$
\mathbf{H} \triangleq\left[\begin{array}{c}
\mathbf{H}_{2} \mathbf{G H}_{1} \\
\mathbf{H}_{0}
\end{array}\right]
$$

and

$$
\mathbf{n}=\left[\begin{array}{c}
\mathbf{H}_{2} \mathbf{G n}_{1}+\mathbf{n}_{2} \\
\mathbf{n}_{0}
\end{array}\right]
$$

where $\mathbf{H} \in \mathbb{C}^{2 N_{D} \times N_{S}}$ is the equivalent MIMO channel matrix, and $\mathbf{n} \in \mathbb{C}^{2 N_{D} \times 1}$ represents the equivalent noise vector. Inserting (4) and (5) into (3), the signal model for the AF MIMO relay system can be written as

$$
\mathbf{y}=\mathbf{H x}+\mathbf{n}
$$

Consider a scenario that the destination node is moving rapidly [13], so the channel is correlated at the transmitter and is uncorrelated at the receiver for the relay-destination link and the direct source-destination link. This model is appropriate for an environment where the destinations is fully surrounded by local scatters [16]. With this assumption, the channel matrices $\mathbf{H}_{0}$ and $\mathbf{H}_{2}$ can be modeled as [13] and [15]

$$
\begin{aligned}
& \mathbf{H}_{0}=\mathbf{H}_{\omega_{0}} \boldsymbol{\Sigma}_{0}^{1 / 2} \\
& \mathbf{H}_{2}=\mathbf{H}_{\omega_{2}} \boldsymbol{\Sigma}_{2}^{1 / 2}
\end{aligned}
$$

where $\mathbf{H}_{\omega_{0}} \in \mathbb{C}^{N_{D} \times N_{S}}$ and $\mathbf{H}_{\omega_{2}} \in \mathbb{C}^{N_{D} \times N_{R}}$ are Gaussian matrices having i.i.d. circularly symmetric complex entries, $\boldsymbol{\Sigma}_{0}$ an $N_{S} \times N_{S}$ covariance matrix of $\mathbf{H}_{0}$ and $\boldsymbol{\Sigma}_{2}$ an $N_{R} \times N_{R}$ covariance matrix of $\mathbf{H}_{2}$ at the relay side. Here, we assume that the destination node feedbacks the two covariances matrices, $\boldsymbol{\Sigma}_{0}$ and $\boldsymbol{\Sigma}_{2}$, to the relay node.

A linear receiver $\mathbf{W}$ is applied at the destination to reduce implementation complexity. The estimated signal at the destination link can be written as

$$
\tilde{\mathbf{x}}=\mathbf{W} \mathbf{y}=\mathbf{W H x}+\mathbf{W} \mathbf{n}
$$

Since the transmitted signal from the relay is $\mathbf{G y}_{1}=$ $\mathbf{G H}_{1} \mathbf{x}+\mathbf{G n}_{1}$, the power constraint on the relay can be expressed as [4]

$$
p(\mathbf{G})=\operatorname{tr}\left\{\mathbf{G}\left(\sigma_{x}^{2} \mathbf{H}_{1} \mathbf{H}_{1}^{H}+\sigma_{1}^{2} \mathbf{I}_{N_{R}}\right) \mathbf{G}^{H}\right\} \leq P_{r}
$$

where $\operatorname{tr}\{$.$\} denotes the trace of a matrix and P_{r}$ is the upper bounded average power used by the relay. Now, our goal is to obtain $\mathbf{G}$ and $\mathbf{W}$ to minimize the MSE of the estimated signal at the destination node. Using the precoder matrix $G$ and the linear receiver $\mathrm{W}$, the MSE function of the estimated signal can be written as [17]

$$
J(\mathbf{G}, \mathbf{W})=\operatorname{tr}\left\{E\left[(\tilde{\mathbf{x}}-\mathbf{x})(\tilde{\mathbf{x}}-\mathbf{x})^{H}\right]\right\} .
$$

Mathematically, the design problem to be considered in the paper can be formulated as

$$
(\mathbf{G}, \mathbf{W})=\underset{(\mathbf{G}, \mathbf{W})}{\arg \min } J(\mathbf{G}, \mathbf{W}), \quad \text { s.t. } p(\mathbf{G}) \leq P_{r} .
$$


After substituting (8) into (10), the MSE function (10) is simplified to

$$
\begin{aligned}
J(\mathbf{G}, \mathbf{W})= & \operatorname{tr}\left\{\sigma_{x}^{2}\left(\mathbf{W H}-\mathbf{I}_{N_{S}}\right)\left(\mathbf{W H}-\mathbf{I}_{N_{S}}\right)^{H}\right. \\
& \left.+\mathbf{W R}_{n} \mathbf{W}^{H}\right\}
\end{aligned}
$$

where $\mathbf{R}_{n}$ is the equivalent noise covariance matrix, given by

$$
\mathbf{R}_{n}=E\left[\mathbf{n n}^{H}\right] \text {. }
$$

Substituting (5) into (13), the noise covariance matrix $\mathbf{R}_{n}$ is given by

$$
\begin{aligned}
\mathbf{R}_{n} & =E\left[\left[\begin{array}{c}
\mathbf{H}_{2} \mathbf{G} \mathbf{n}_{1}+\mathbf{n}_{2} \\
\mathbf{n}_{0}
\end{array}\right]\left[\begin{array}{c}
\mathbf{H}_{2} \mathbf{G} \mathbf{n}_{1}+\mathbf{n}_{2} \\
\mathbf{n}_{0}
\end{array}\right]\right. \\
& =\left[\begin{array}{ll}
\sigma_{1}^{2} \mathbf{H}_{2} \mathbf{G G}^{H} \mathbf{H}_{2}^{H}+\sigma_{2}^{2} \mathbf{I}_{N_{D}} & \mathbf{0}_{N_{D} \times N_{D}} \\
\mathbf{0}_{N_{D} \times N_{D}} & \mathbf{I}_{N_{D}}
\end{array}\right] .
\end{aligned}
$$

Note that the constrained optimization problem (11) is not easy to solve directly due to the fact that the optimization function $J(\mathbf{G}, \mathbf{W})$ is a non-linear and non-convex function of $\mathbf{G}$ and $\mathbf{W}$ and the power constraint is non-linear function of G. In the following sections an iterative based optimal covariance algorithm and suboptimal covariance algorithm will be proposed to solve the constrained non-linear optimization problem.

\section{Optimal Precoder Design}

For any given precoding matrix $\mathbf{G}$ which satisfies the power constraint (9), the optimal linear receiver $\mathbf{W}$ that minimizes the MSE function $J(\mathbf{G}, \mathbf{W})$ is the MMSE (Wiener) receiver [17], which is given by

$$
\mathbf{W}=\sigma_{x}^{2} \mathbf{H}^{H}\left(\sigma_{x}^{2} \mathbf{H} \mathbf{H}^{H}+\mathbf{R}_{n}\right)^{-1} .
$$

After substituting (15) into (12), the MSE function is obtained as

$$
J(\mathbf{G})=\sigma_{x}^{2} \operatorname{tr}\left\{\mathbf{I}_{N_{S}}-\sigma_{x}^{2} \mathbf{H}^{H}\left(\sigma_{x}^{2} \mathbf{H} \mathbf{H}^{H}+\mathbf{R}_{n}\right)^{-1} \mathbf{H}\right\} .
$$

Using the following matrix inversion lemma [17]

$$
\begin{aligned}
(\mathbf{A}+\mathbf{B C D})^{-1}= & \mathbf{A}^{-1}-\mathbf{A}^{-1} \mathbf{B} \\
& \times\left(\mathbf{D A} \mathbf{A}^{-1} \mathbf{B}+\mathbf{C}^{-1}\right)^{-1} \mathbf{D} \mathbf{A}^{-1},
\end{aligned}
$$

the MSE function (16) can be written as

$$
J(\mathbf{G})=\sigma_{x}^{2} \operatorname{tr}\left\{\left[\mathbf{I}_{N_{S}}+\sigma_{x}^{2} \mathbf{H}^{H} \mathbf{R}_{n}^{-1} \mathbf{H}\right]^{-1}\right\} .
$$

Substituting (4) and (14) into (18), the MSE function can be expressed as

$$
\begin{aligned}
J(\mathbf{G})= & \sigma_{x}^{2} \operatorname{tr}\left\{\left[\mathbf{I}_{N_{S}}+\sigma_{x}^{2} \mathbf{H}_{0}^{H} \mathbf{H}_{0}+\sigma_{x}^{2} \mathbf{H}_{1}^{H} \mathbf{G}^{H} \mathbf{H}_{2}^{H}\right.\right. \\
& \left.\left.\times\left(\sigma_{1}^{2} \mathbf{H}_{2} \mathbf{G} \mathbf{G}^{H} \mathbf{H}_{2}^{H}+\sigma_{2}^{2} \mathbf{I}_{N_{D}}\right)^{-1} \mathbf{H}_{2} \mathbf{G} \mathbf{H}_{1}\right]^{-1}\right\} .
\end{aligned}
$$

Using the matrix inversion lemma (17), the MSE function (19) can be written as

$$
\begin{aligned}
J(\mathbf{G})= & \sigma_{x}^{2} \operatorname{tr}\left\{\left[\mathbf{I}_{N_{S}}+\sigma_{x}^{2} \mathbf{H}_{0}^{H} \mathbf{H}_{0}+\frac{\sigma_{x}^{2}}{\sigma_{1}^{2}} \mathbf{H}_{1}^{H}\left[\mathbf{I}_{N_{R}}\right.\right.\right. \\
& \left.\left.\left.-\left(\mathbf{I}_{N_{R}}+\frac{\sigma_{1}^{2}}{\sigma_{2}^{2}} \mathbf{G}^{H} \mathbf{H}_{2}^{H} \mathbf{H}_{2} \mathbf{G}\right)^{-1}\right] \mathbf{H}_{1}\right]^{-1}\right\}
\end{aligned}
$$

Now the problem is reduced to find the optimal $\mathbf{G}$ that minimize $J(\mathbf{G})$ subject to the power constraint (9). We introduce the eigenvalue decomposition of $\boldsymbol{\Sigma}_{0}$ as

$$
\boldsymbol{\Sigma}_{0}=\mathbf{V}_{\Sigma_{0}} \boldsymbol{\Lambda}_{\Sigma_{0}} \mathbf{V}_{\Sigma_{0}}^{H}
$$

where $\boldsymbol{\Lambda}_{\Sigma_{0}}=\operatorname{diag}\left\{\Lambda_{\Sigma_{0}, 1} \cdots \Lambda_{\Sigma_{0}, N_{S}}\right\}$ with $\Lambda_{\Sigma_{0}, 1} \geq \cdots \geq$ $\Lambda_{\Sigma_{0}, N_{S}}$. The columns of $\mathbf{V}_{\Sigma_{0}}$ are the eigenvectors of $\boldsymbol{\Sigma}_{0}$ for the corresponding eigenvalues. Substituting (21) into (7), the channel matrix $\mathbf{H}_{0}$ can be written as

$$
\mathbf{H}_{0} \triangleq \widetilde{\mathbf{H}}_{\omega_{0}} \boldsymbol{\Lambda}_{\Sigma_{0}}^{1 / 2} \mathbf{V}_{\Sigma_{0}}^{H}
$$

where $\widetilde{\mathbf{H}}_{\omega_{0}} \triangleq \mathbf{H}_{\omega_{0}} \mathbf{V}_{\Sigma_{0}}$ has the same distribution as $\mathbf{H}_{\omega_{0}}$, because the unitary matrix $\mathbf{V}_{\Sigma_{0}}$ does not change the statistical distribution of $\mathbf{H}_{\omega_{0}}$. The singular value decomposition (SVD) of $\mathbf{H}_{1}$ can be expressed as

$$
\mathbf{H}_{1}=\mathbf{U}_{1} \boldsymbol{\Lambda}_{1}^{1 / 2} \mathbf{V}_{1}^{H}
$$

where $\boldsymbol{\Lambda}_{1}=\operatorname{diag}\left\{\Lambda_{1,1} \cdots \Lambda_{1, R_{1}}\right\}$ is a diagonal matrix with $\Lambda_{1,1} \geq \cdots \geq \Lambda_{1, R_{1}}, R_{1}=\min \left(N_{S}, N_{R}\right)$, and the dimensions of $\mathbf{U}_{1}$ and $\mathbf{V}_{1}$ are $N_{R} \times R_{1}, N_{S} \times R_{1}$, respectively. Now we introduce the eigenvalue decomposition of $\boldsymbol{\Sigma}_{2}$ as

$$
\boldsymbol{\Sigma}_{2}=\mathbf{V}_{\Sigma_{2}} \Lambda_{\Sigma_{2}} \mathbf{V}_{\Sigma_{2}}^{H}
$$

where $\boldsymbol{\Lambda}_{\Sigma_{2}}=\operatorname{diag}\left\{\Lambda_{\Sigma_{2}, 1} \cdots \Lambda_{\Sigma_{2}, N_{R}}\right\}$ with $\Lambda_{\Sigma_{2}, 1} \geq \cdots \geq$ $\Lambda_{\Sigma_{2}, N_{R}}$. The columns of $\mathbf{V}_{\Sigma_{2}}$ are the eigenvectors of $\Sigma_{2}$ for the corresponding eigenvalues. Substituting (24) into (7), the channel matrix $\mathbf{H}_{2}$ can be rewritten as

$$
\mathbf{H}_{2} \triangleq \widetilde{\mathbf{H}}_{\omega_{2}} \boldsymbol{\Lambda}_{\Sigma_{2}}^{1 / 2} \mathbf{V}_{\Sigma_{2}}^{H}
$$

where $\widetilde{\mathbf{H}}_{\omega_{2}} \triangleq \mathbf{H}_{\omega_{2}} \mathbf{V}_{\Sigma_{2}}$ has the same distribution as $\mathbf{H}_{\omega_{2}}$. Let's assume that the optimal precoding matrix $\mathbf{G}$ which minimizes (20) can be expressed as

$$
\mathbf{G}=\mathbf{V}_{\Sigma_{2}} \widetilde{\mathbf{G}} \mathbf{U}_{1}^{H}
$$

Substituting (22)-(26) into (20), now the MSE function is given by

$$
\begin{aligned}
J(\widetilde{\mathbf{G}})= & \sigma_{x}^{2} \operatorname{tr}\left\{\left[\mathbf{I}_{N_{S}}+\sigma_{x}^{2} \mathbf{V}_{\Sigma_{0}} \boldsymbol{\Lambda}_{\Sigma_{0}}^{1 / 2} \widetilde{\mathbf{H}}_{\omega_{0}}^{H} \widetilde{\mathbf{H}}_{\omega_{0}} \boldsymbol{\Lambda}_{\Sigma_{0}}^{1 / 2} \mathbf{V}_{\Sigma_{0}}^{H}\right.\right. \\
& \left.\left.+\frac{\sigma_{x}^{2}}{\sigma_{1}^{2}} \mathbf{V}_{1} \boldsymbol{\Lambda}_{1}^{1 / 2} \mathbf{U}_{1}^{H}\left[\mathbf{I}_{N_{R}}-\mathbf{D}_{1}\right] \mathbf{U}_{1} \boldsymbol{\Lambda}_{1}^{1 / 2} \mathbf{V}_{1}^{H}\right]^{-1}\right\}
\end{aligned}
$$

where

$$
\mathbf{D}_{1}=\left(\mathbf{I}_{N_{R}}+\frac{\sigma_{1}^{2}}{\sigma_{2}^{2}} \mathbf{U}_{1} \widetilde{\mathbf{G}}^{H} \boldsymbol{\Lambda}_{\Sigma_{2}}^{1 / 2} \widetilde{\mathbf{H}}_{\omega_{2}}^{H} \widetilde{\mathbf{H}}_{\omega_{2}} \boldsymbol{\Lambda}_{\Sigma_{2}}^{1 / 2} \widetilde{\mathbf{G}} \mathbf{U}_{1}^{H}\right)^{-1}
$$

Since $\mathbf{U}_{1}^{H} \mathbf{U}_{1}=\mathbf{I}_{R_{1}}$, the MSE function (27) can be simplified to

$$
\begin{aligned}
J(\widetilde{\mathbf{G}})= & \sigma_{x}^{2} \operatorname{tr}\left\{\left[\mathbf{I}_{N_{S}}+\sigma_{x}^{2} \mathbf{V}_{\Sigma_{0}} \boldsymbol{\Lambda}_{\Sigma_{0}}^{1 / 2} \widetilde{\mathbf{H}}_{\omega_{0}}^{H} \widetilde{\mathbf{H}}_{\omega_{0}} \boldsymbol{\Lambda}_{\Sigma_{0}}^{1 / 2} \mathbf{V}_{\Sigma_{0}}^{H}\right.\right. \\
& \left.\left.+\frac{\sigma_{x}^{2}}{\sigma_{1}^{2}}\left(\mathbf{V}_{1} \boldsymbol{\Lambda}_{1} \mathbf{V}_{1}^{H}-\mathbf{V}_{1} \boldsymbol{\Lambda}_{1}^{1 / 2} \mathbf{D}_{2} \boldsymbol{\Lambda}_{1}^{1 / 2} \mathbf{V}_{1}^{H}\right)\right]^{-1}\right\}
\end{aligned}
$$

where

$$
\mathbf{D}_{2}=\left(\mathbf{I}_{R_{1}}+\frac{\sigma_{1}^{2}}{\sigma_{2}^{2}} \widetilde{\mathbf{G}}^{H} \boldsymbol{\Lambda}_{\Sigma_{2}}^{1 / 2} \widetilde{\mathbf{H}}_{\omega_{2}}^{H} \widetilde{\mathbf{H}}_{\omega_{2}} \boldsymbol{\Lambda}_{\Sigma_{2}}^{1 / 2} \widetilde{\mathbf{G}}\right)^{-1}
$$


It can be seen from (28) that $J(\widetilde{\mathbf{G}})$ depends on $\widetilde{\mathbf{H}}_{\omega_{0}}$ and $\widetilde{\mathbf{H}}_{\omega_{2}}$, which are random and unknown. In the following, we optimize $E_{\mathbf{H}_{\omega_{0,2}}}[J(\widetilde{\mathbf{G}})]$, where $E_{\mathbf{H}_{\omega_{0,2}}}[$.$] indicates that the$ expectation is taken with respect to the random matrices $\widetilde{\mathbf{H}}_{\omega_{0}}$ and $\widetilde{\mathbf{H}}_{\omega_{2}}$. Now $E_{\mathbf{H}_{\omega_{0,2}}}[J(\widetilde{\mathbf{G}})]$ can be expressed as

$$
\begin{aligned}
E_{\mathbf{H}_{\omega_{0}, 2}}[J(\widetilde{\mathbf{G}})]= & \sigma_{x}^{2} \sigma_{1}^{2} E_{\mathbf{H}_{\omega_{0,2}}}\left[\operatorname { t r } \left\{\left[\sigma_{1}^{2} \mathbf{I}_{N_{S}}+\sigma_{x}^{2} \sigma_{1}^{2} \mathbf{V}_{\Sigma_{0}} \boldsymbol{\Lambda}_{\Sigma_{0}}^{1 / 2}\right.\right.\right. \\
& \times \widetilde{\mathbf{H}}_{\omega_{0}}^{H} \widetilde{\mathbf{H}}_{\omega_{0}} \boldsymbol{\Lambda}_{\Sigma_{0}}^{1 / 2} \mathbf{V}_{\Sigma_{0}}^{H}+\sigma_{x}^{2} \mathbf{V}_{1} \boldsymbol{\Lambda}_{1} \mathbf{V}_{1}^{H} \\
& \left.\left.\left.-\sigma_{x}^{2} \mathbf{V}_{1} \boldsymbol{\Lambda}_{1}^{1 / 2} \mathbf{D}_{2} \boldsymbol{\Lambda}_{1}^{1 / 2} \mathbf{V}_{1}^{H}\right]^{-1}\right\}\right]
\end{aligned}
$$

Now the work is left to determine $\widetilde{\mathbf{G}}$ of precoder matrix G. The optimal precoder allocates power according to the eigenmodes of $\mathbf{H}_{1} \mathbf{H}_{1}^{H}, \boldsymbol{\Sigma}_{0}$ and $\boldsymbol{\Sigma}_{2}$.

Direct minimization of (29) for the optimal power allocation is difficult. In the following, the lower bound of the MSE is used together with the power constraint (9) to derive the optimal power allocation for the precoder matrix G. Since $J(\widetilde{\mathbf{G}})$ is convex in $\widetilde{\mathbf{H}}_{\omega_{0}}^{H} \widetilde{\mathbf{H}}_{\omega_{0}}$ and $\widetilde{\mathbf{H}}_{\omega_{2}}^{H} \widetilde{\mathbf{H}}_{\omega_{2}}$, which is proved in Appendix, we use Jensen's inequality [18] to derive the following lower bound

$$
\begin{aligned}
& J_{L}(\widetilde{\mathbf{G}})=\sigma_{x}^{2} \sigma_{1}^{2} \operatorname{tr}\left\{\left[\sigma_{1}^{2} \mathbf{I}_{N_{S}}+\sigma_{x}^{2} \sigma_{1}^{2} \mathbf{V}_{\Sigma_{0}} \boldsymbol{\Lambda}_{\Sigma_{0}}^{1 / 2} E_{\mathbf{H}_{\omega_{0}}}\left[\widetilde{\mathbf{H}}_{\omega_{0}}^{H} \widetilde{\mathbf{H}}_{\omega_{0}}\right]\right.\right. \\
& \left.\left.\times \boldsymbol{\Lambda}_{\Sigma_{0}}^{1 / 2} \mathbf{V}_{\Sigma_{0}}^{H}+\sigma_{x}^{2} \mathbf{V}_{1} \boldsymbol{\Lambda}_{1} \mathbf{V}_{1}^{H}-\sigma_{x}^{2} \mathbf{V}_{1} \boldsymbol{\Lambda}_{1}^{1 / 2} \mathbf{D}_{3} \boldsymbol{\Lambda}_{1}^{1 / 2} \mathbf{V}_{1}^{H}\right]^{-1}\right\}
\end{aligned}
$$

where

$$
\mathbf{D}_{3}=\left(\mathbf{I}_{R_{1}}+\frac{\sigma_{1}^{2}}{\sigma_{2}^{2}} \widetilde{\mathbf{G}}^{H} \boldsymbol{\Lambda}_{\Sigma_{2}}^{1 / 2} E_{\mathbf{H}_{\omega_{2}}}\left[\widetilde{\mathbf{H}}_{\omega_{2}}^{H} \widetilde{\mathbf{H}}_{\omega_{2}}\right] \boldsymbol{\Lambda}_{\Sigma_{2}}^{1 / 2} \widetilde{\mathbf{G}}\right)^{-1} .
$$

Now the MSE function is simplified to

$$
\begin{aligned}
J_{L}(\widetilde{\mathbf{G}})= & \sigma_{x}^{2} \sigma_{1}^{2} \operatorname{tr}\left\{\left[\sigma_{1}^{2} \mathbf{I}_{N_{S}}+\sigma_{x}^{2} \sigma_{1}^{2} N_{D} \mathbf{V}_{\Sigma_{0}} \boldsymbol{\Lambda}_{\Sigma_{0}} \mathbf{V}_{\Sigma_{0}}^{H}\right.\right. \\
& \left.\left.+\sigma_{x}^{2} \mathbf{V}_{1} \boldsymbol{\Lambda}_{1} \mathbf{V}_{1}^{H}-\sigma_{x}^{2} \mathbf{V}_{1} \boldsymbol{\Lambda}_{1}^{1 / 2} \mathbf{D}_{4} \boldsymbol{\Lambda}_{1}^{1 / 2} \mathbf{V}_{1}^{H}\right]^{-1}\right\}(30)
\end{aligned}
$$

where

$$
\mathbf{D}_{4}=\left(\mathbf{I}_{R_{1}}+\frac{\sigma_{1}^{2} N_{D}}{\sigma_{2}^{2}} \widetilde{\mathbf{G}}^{H} \boldsymbol{\Lambda}_{\Sigma_{2}} \widetilde{\mathbf{G}}\right)^{-1}
$$

Here we used $E_{\mathbf{H}_{\omega_{\mathbf{0}}}}\left[\widetilde{\mathbf{H}}_{\omega_{0}}^{H} \widetilde{\mathbf{H}}_{\omega_{0}}\right]=E_{\mathbf{H}_{\omega_{2}}}\left[\widetilde{\mathbf{H}}_{\omega_{2}}^{H} \widetilde{\mathbf{H}}_{\omega_{2}}\right]=$ $N_{D} \mathbf{I}_{N_{R}}$. Substituting (23) and (26) into (9), the power constraint for the relay node can be expressed as

$$
\begin{aligned}
p(\widetilde{\mathbf{G}})= & t r\left\{\mathbf{V}_{\Sigma_{2}} \widetilde{\mathbf{G}} \mathbf{U}_{1}^{H}\left(\sigma_{x}^{2} \mathbf{U}_{1} \boldsymbol{\Lambda}_{1} \mathbf{U}_{1}^{H}+\sigma_{1}^{2} \mathbf{I}_{N_{R}}\right)\right. \\
& \left.\times \mathbf{U}_{1} \widetilde{\mathbf{G}}^{H} \mathbf{V}_{\Sigma_{2}}^{H}\right\} \leq P_{r} .
\end{aligned}
$$

Using the SVD and trace properties, the power constraint (31) can be simplified to

$$
p(\widetilde{\mathbf{G}})=\operatorname{tr}\left\{\widetilde{\mathbf{G}}\left(\sigma_{x}^{2} \boldsymbol{\Lambda}_{1}+\sigma_{1}^{2} \mathbf{I}_{R_{1}}\right) \widetilde{\mathbf{G}}^{H}\right\} \leq P_{r} .
$$

The remaining task is to optimize $\widetilde{\mathbf{G}}$. From (30) and (32), we have the following constrained optimization problem.

$$
\begin{gathered}
\min J_{L}(\widetilde{\mathbf{G}})=\sigma_{x}^{2} \sigma_{1}^{2} \operatorname{tr}\left\{\left[\sigma_{1}^{2} \mathbf{I}_{N_{S}}+\sigma_{x}^{2} \sigma_{1}^{2} N_{D} \mathbf{V}_{\Sigma_{0}} \boldsymbol{\Lambda}_{\Sigma_{0}} \mathbf{V}_{\Sigma_{0}}^{H}\right.\right. \\
\left.\left.+\sigma_{x}^{2} \mathbf{V}_{1} \boldsymbol{\Lambda}_{1} \mathbf{V}_{1}^{H}-\sigma_{x}^{2} \mathbf{V}_{1} \boldsymbol{\Lambda}_{1}^{1 / 2} \mathbf{D}_{4} \boldsymbol{\Lambda}_{1}^{1 / 2} \mathbf{V}_{1}^{H}\right]^{-1}\right\} \\
\text { s.t. } p(\widetilde{\mathbf{G}})=\operatorname{tr}\left\{\widetilde{\mathbf{G}}\left(\sigma_{x}^{2} \boldsymbol{\Lambda}_{1}+\sigma_{1}^{2} \mathbf{I}_{R_{1}}\right) \widetilde{\mathbf{G}}^{H}\right\} \leq P_{r}
\end{gathered}
$$

\section{A. Optimal Covariance Algorithm}

The constrained optimization problem (33)-(34) does not have a closed-form solution due to the presence of the direct link channel $\mathbf{H}_{0}$. The problem (33)-(34) can be solved by resorting to numerical methods, such as the projected gradient algorithm [19].

The relay precoding matrix $\widetilde{\mathbf{G}}$ is optimized by solving the following constrained optimization problem

$$
\begin{aligned}
& \min J_{L}(\tilde{\mathbf{G}})=\sigma_{x}^{2} \sigma_{1}^{2} \operatorname{tr}\left\{\left[\mathbf{B}-\mathbf{C D}_{4} \mathbf{C}^{H}\right]^{-1}\right\} \\
& \text { s.t. } p(\tilde{\mathbf{G}})=\operatorname{tr}\left\{\widetilde{\mathbf{G}} \mathbf{M} \widetilde{\mathbf{G}}^{H}\right\} \leq P_{r}
\end{aligned}
$$

where

$$
\begin{aligned}
\mathbf{B} & =\sigma_{1}^{2} \mathbf{I}_{N_{S}}+\sigma_{x}^{2} \sigma_{1}^{2} N_{D} \mathbf{V}_{\Sigma_{0}} \boldsymbol{\Lambda}_{\Sigma_{0}} \mathbf{V}_{\Sigma_{0}}^{H}+\sigma_{x}^{2} \mathbf{V}_{1} \boldsymbol{\Lambda}_{1} \mathbf{V}_{1}^{H} \\
\mathbf{C} & =\sigma_{x} \mathbf{V}_{1} \boldsymbol{\Lambda}_{1}^{1 / 2} \\
\mathbf{M} & =\sigma_{x}^{2} \boldsymbol{\Lambda}_{1}+\sigma_{1}^{2} \mathbf{I}_{R_{1}}
\end{aligned}
$$

The gradient of (35) is given by

$$
\begin{aligned}
\nabla J_{L}(\tilde{\mathbf{G}})= & \frac{-2 \sigma_{1}^{2} N_{D}}{\sigma_{2}^{2}}\left[\mathbf{D}_{4} \mathbf{C}^{H}\left(\mathbf{B}-\mathbf{C D}_{4} \mathbf{C}^{H}\right)^{-2}\right. \\
& \left.\times \mathbf{C D}_{4} \widetilde{\mathbf{G}}^{H} \boldsymbol{\Lambda}_{\Sigma_{2}}\right]^{H}
\end{aligned}
$$

where the derivatives of $\partial \operatorname{tr}\left(\boldsymbol{\Theta} \mathbf{X}^{-1}\right) / \partial \mathbf{X}=-\left(\mathbf{X}^{-1} \mathbf{\Theta} \mathbf{X}^{-1}\right)^{T}$ and $\partial \operatorname{tr}(\mathbf{\Theta X}) / \partial \mathbf{X}=\boldsymbol{\Theta}^{T}$ are used to obtain (37). The problem (35)-(36) can be solved by the projected gradient algorithm to optimize the matrix elements of $\widetilde{\mathbf{G}}$.

\section{B. Suboptimal Covariance Algorithm}

Now we propose a relay matrix design algorithm which is suboptimal, but has a significant computational complexity reduction compared with the gradient projection-based optimal design. Similar to [6]-[15], let us assume that the matrix $\widetilde{\mathbf{G}}=$ $\left[\boldsymbol{\Lambda}_{G}^{1 / 2}, \mathbf{0}_{R_{1} \times\left(N_{R}-R_{1}\right)}\right]^{T}$, where $\boldsymbol{\Lambda}_{G}=\operatorname{diag}\left\{\Lambda_{G, 1} \cdots \Lambda_{G, R_{1}}\right\}$. Hence, the equation (26) can be rewritten as

$$
\mathbf{G}=\overline{\mathbf{V}}_{\Sigma_{2}} \Lambda_{G}^{1 / 2} \mathbf{U}_{1}^{H}
$$

where $\overline{\mathbf{V}}_{\Sigma_{2}}$ is a matrix taking the first $R_{1}$ columns of $\mathbf{V}_{\Sigma_{2}}$. Then, the constrained optimization problem is reduced to

$$
\begin{array}{cl}
\min & J_{L}\left(\boldsymbol{\Lambda}_{G}\right)=\sigma_{x}^{2} \sigma_{1}^{2} \operatorname{tr}\left\{\left[\sigma_{1}^{2} \mathbf{I}_{N_{S}}+\sigma_{x}^{2} \sigma_{1}^{2} N_{D} \mathbf{V}_{\Sigma_{0}} \boldsymbol{\Lambda}_{\Sigma_{0}} \mathbf{V}_{\Sigma_{0}}^{H}\right.\right. \\
& \left.\left.+\sigma_{x}^{2} \mathbf{V}_{1} \boldsymbol{\Lambda}_{1}\left[\mathbf{I}_{R_{1}}-\left(\mathbf{I}_{R_{1}}+\frac{\sigma_{1}^{2} N_{D}}{\sigma_{2}^{2}} \boldsymbol{\Lambda}_{G} \overline{\boldsymbol{\Lambda}}_{\Sigma_{2}}\right)^{-1}\right] \mathbf{V}_{1}^{H}\right]^{-1}\right\} \\
\text { s.t. } & p\left(\boldsymbol{\Lambda}_{G}\right)=\operatorname{tr}\left\{\left(\sigma_{x}^{2} \boldsymbol{\Lambda}_{1}+\sigma_{1}^{2} \mathbf{I}_{R_{1}}\right) \boldsymbol{\Lambda}_{G}\right\} \leq P_{r}
\end{array}
$$

where $\bar{\Lambda}_{\Sigma_{2}}=\operatorname{diag}\left\{\Lambda_{\Sigma_{2}, 1} \cdots \Lambda_{\Sigma_{2}, R_{1}}\right\}$.

To proceed further, let us use the matrix inversion lemma (17) to rewrite the MSE function (39) as

$$
\begin{aligned}
J_{L}\left(\boldsymbol{\Lambda}_{G}\right)= & \sigma_{x}^{2} \operatorname{tr}\left\{\left[\mathbf{I}_{N_{S}}+\sigma_{x}^{2} N_{D} \mathbf{V}_{\Sigma_{0}} \boldsymbol{\Lambda}_{\Sigma_{0}} \mathbf{V}_{\Sigma_{0}}^{H}+\frac{\sigma_{x}^{2} N_{D}}{\sigma_{2}^{2}} \mathbf{V}_{1} \boldsymbol{\Lambda}_{1}\right.\right. \\
& \left.\left.\times \boldsymbol{\Lambda}_{G} \overline{\boldsymbol{\Lambda}}_{\Sigma_{2}}\left(\mathbf{I}_{R_{1}}+\frac{\sigma_{1}^{2} N_{D}}{\sigma_{2}^{2}} \overline{\boldsymbol{\Lambda}}_{\Sigma_{2}} \boldsymbol{\Lambda}_{G}\right)^{-1} \mathbf{V}_{1}^{H}\right]^{-1}\right\} .(41)
\end{aligned}
$$


We consider an upper-bound of (41) as follows. By introducing $\mathbf{E}_{1}=\frac{\sigma_{x}^{2} N_{D}}{\sigma_{2}^{2}} \boldsymbol{\Lambda}_{1} \boldsymbol{\Lambda}_{G} \overline{\boldsymbol{\Lambda}}_{\Sigma_{2}}\left(\mathbf{I}_{R_{1}}+\frac{\sigma_{1}^{2} N_{D}}{\sigma_{2}^{2}} \overline{\boldsymbol{\Lambda}}_{\Sigma_{2}} \boldsymbol{\Lambda}_{G}\right)^{-1}$, the MSE function (41) can be written as

$J_{L}\left(\boldsymbol{\Lambda}_{G}\right)=\sigma_{x}^{2} \operatorname{tr}\left\{\left[\mathbf{I}_{N_{S}}+\mathbf{V}_{1} \mathbf{E}_{1} \mathbf{V}_{1}^{H}+\sigma_{x}^{2} N_{D} \mathbf{V}_{\Sigma_{0}} \boldsymbol{\Lambda}_{\Sigma_{0}} \mathbf{V}_{\Sigma_{0}}^{H}\right]_{(42)}^{-1}\right\}$.

Let us introduce $\overline{\mathbf{V}}_{1}=\left[\mathbf{V}_{1}, \mathbf{V}_{1}^{\perp}\right]$ such that $\overline{\mathbf{V}}_{1}$ is an $N_{s} \times N_{s}$ unitary matrix. Obviously, if $R_{1}=N_{s}, \overline{\mathbf{V}}_{1}=\mathbf{V}_{1}$. Then (42) can be equivalently rewritten as

$$
J_{L}\left(\boldsymbol{\Lambda}_{G}\right)=\sigma_{x}^{2} \operatorname{tr}\left\{\left[\mathbf{A}+\sigma_{x}^{2} N_{D} \overline{\mathbf{V}}_{1}^{H} \mathbf{V}_{\Sigma_{0}} \boldsymbol{\Lambda}_{\Sigma_{0}} \mathbf{V}_{\Sigma_{0}}^{H} \overline{\mathbf{V}}_{1}\right]^{-1}\right\}
$$

where

$$
\mathbf{A}=\mathbf{I}_{N_{S}}+\left[\mathbf{I}_{R_{1}}, \mathbf{0}_{R_{1} \times\left(N_{s}-R_{1}\right)}\right]^{T} \mathbf{E}_{1}\left[\mathbf{I}_{R_{1}}, \mathbf{0}_{R_{1} \times\left(N_{s}-R_{1}\right)}\right] .
$$

Applying the matrix inversion lemma (17), the MSE function (43) can be rewritten as

$$
J_{L}\left(\boldsymbol{\Lambda}_{G}\right)=\sigma_{x}^{2}\left[\operatorname{tr}\left(\mathbf{A}^{-1}\right)-\operatorname{tr}\left(\mathbf{A}^{-1}\left(\mathbf{C}+\mathbf{A}^{-1}\right)^{-1} \mathbf{A}^{-1}\right)\right]
$$

where $\mathbf{C}=\sigma_{x}^{2} N_{D}\left(\overline{\mathbf{V}}_{1}^{H} \mathbf{V}_{\Sigma_{0}} \boldsymbol{\Lambda}_{\Sigma_{0}} \mathbf{V}_{\Sigma_{0}}^{H} \overline{\mathbf{V}}_{1}\right)^{-1}$. By using the following inequality from [11]

$$
\begin{aligned}
& \operatorname{tr}\left(\mathbf{A}^{-1}\left(\mathbf{C}+\mathbf{A}^{-1}\right)^{-1} \mathbf{A}^{-1}\right) \\
& \geq \operatorname{tr}\left(\mathbf{A}^{-1}\left(\operatorname{diag}(\mathbf{C})+\mathbf{A}^{-1}\right)^{-1} \mathbf{A}^{-1}\right)
\end{aligned}
$$

an upper-bound of $J_{L}\left(\boldsymbol{\Lambda}_{G}\right)$ is given by

$$
J_{U}\left(\boldsymbol{\Lambda}_{G}\right)=\sigma_{x}^{2}\left[\operatorname{tr}\left(\mathbf{A}^{-1}\right)-\operatorname{tr}\left(\mathbf{A}^{-1}\left(\operatorname{diag}(\mathbf{C})+\mathbf{A}^{-1}\right)^{-1} \mathbf{A}^{-1}\right)\right] .
$$

From (46), the diagonal elements of $\Lambda_{\mathrm{G}}$ can be obtained by solving the following optimization problem with scalar variables

$$
\begin{aligned}
\min _{\left\{\Lambda_{G, i}\right\}} & \sum_{i=1}^{R_{1}} \frac{\left(\sigma_{1}^{2} N_{D} \Lambda_{\Sigma_{2}, i} \Lambda_{G, i}+\sigma_{2}^{2}\right) \sigma_{x}^{2} \lambda_{c, i}}{D_{5} \Lambda_{G, i}+\sigma_{2}^{2} \lambda_{c, i}+\sigma_{2}^{2}} \\
\text { s.t. } & \sum_{i=1}^{R_{1}}\left(\sigma_{x}^{2} \Lambda_{1, i}+\sigma_{1}^{2}\right) \Lambda_{G, i} \leq P_{r}
\end{aligned}
$$

where

$$
\begin{gathered}
D_{5}=\left(\sigma_{1}^{2} \lambda_{c, i}+\sigma_{x}^{2} \Lambda_{1, i} \lambda_{c, i}+\sigma_{1}^{2}\right) N_{D} \Lambda_{\Sigma_{2}, i} \\
\lambda_{c, i}=\sigma_{x}^{2} N_{D} \operatorname{diag}\left(\left(\overline{\mathbf{V}}_{1}^{H} \mathbf{V}_{\Sigma_{0}} \boldsymbol{\Lambda}_{\Sigma_{0}} \mathbf{V}_{\Sigma_{0}}^{H} \overline{\mathbf{V}}_{1}\right)^{-1}\right)
\end{gathered}
$$

Using the Karush-Kuhn-Tucker (KKT) conditions [19], the optimal diagonal elements of $\Lambda_{G, i}$ are obtained as

$$
\Lambda_{G, i}=\frac{1}{D_{5}}\left(\sqrt{\frac{\sigma_{x}^{4} \sigma_{2}^{2} N_{D} \Lambda_{1, i} \Lambda_{\Sigma_{2}, i} \lambda_{c, i}^{2}}{\mu\left(\sigma_{x}^{2} \Lambda_{1, i}+\sigma_{1}^{2}\right)}}-\sigma_{2}^{2} \lambda_{c, i}-\sigma_{2}^{2}\right)^{+}
$$

where $(x)^{+}=\max (x, 0)$ and $\mu$ should be chosen to meet the power constraint (48).

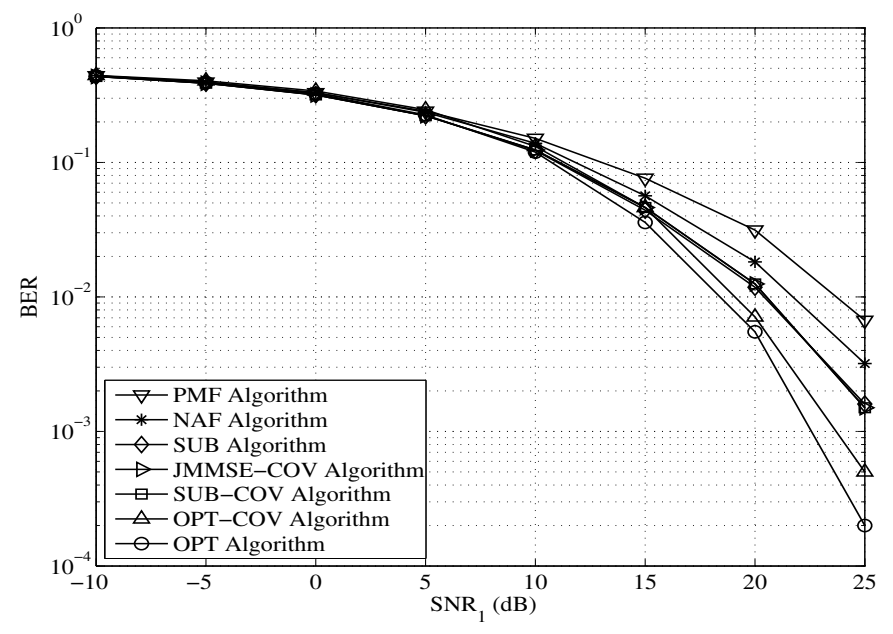

Fig. 2. BER versus $S N R_{1}$ while fixing $N_{S}=N_{R}=N_{D}=4, \triangle_{0}=$ $1^{\circ}, \triangle_{2}=30^{\circ}, S N R_{2}=20 d B, S N R_{0}=S N R_{1}-10 d B$.

\section{Simulation Results}

In this section, we illustrate the performance of the proposed schemes by numerical examples. We simulate the AF MIMO relay system with $N_{S}=N_{R}=N_{D}=4$. The entries of channel matrices $\mathbf{H}_{\omega_{0}}, \mathbf{H}_{1}$ and $\mathbf{H}_{\omega_{2}}$ are generated as complex Gaussian variables with zero mean and unit variances and the symbols are generated from QPSK constellation.

The elements of covariance matrices $\boldsymbol{\Sigma}_{0}$ of $\mathbf{H}_{0}$ and $\boldsymbol{\Sigma}_{2}$ of $\mathbf{H}_{2}$ are generated by $\Sigma_{i, j}=j_{0}(\triangle \pi|i-j|)$ [16], where $j_{0}($.$) is the zeroth order Bessel function of the first kind,$ $\triangle$ the angle of fading spread. We consider the angle spread as $\triangle_{0}=1^{o}$ for the direct source-destination link and $\triangle_{2}=$ $30^{\circ}$ for the relay-destination link. The SNRs for the direct source-destination, the source-relay and relay-destination links are defined as follows $S N R_{0}=\frac{\sigma_{x}^{2}}{\sigma_{0}^{2}}, S N R_{1}=\frac{\sigma_{x}^{2}}{\sigma_{1}^{2}}, S N R_{2}=$ $\frac{P_{r}}{N_{R} \sigma_{2}^{2}}$. We consider a scenario as assumed in section II that the destination node is moving rapidly. Hence, to implement the assumption in simulation, we set the distance between the relay to destination link is fixed, where the source to relay and source to destination distances are varying. For establishing the scenario, we set $S N R_{2}=20 d B, S N R_{0}=S N R_{1}-10 d B$.

We compare the performance of the proposed OPT-COV algorithm with the naive amplify-and-forward (NAF) algorithm [5], the pseudo match-and-forward (PMF) algorithm [5], SUB algorithm [9], the JMMSE-COV algorithm [15], OPT algorithm [9] and the suboptimal covariance (SUB-COV) algorithm. The full CSI scheme, also known as OPT algorithm [9] provides the lower-bound of the proposed scheme. For the proposed OPT-COV algorithm, the projected gradient method is applied to optimize $\widetilde{\mathbf{G}}$ in (35)-(36).

Fig. 2 shows the performance of MSE algorithms in terms of BER versus $S N R_{1}$. It can be seen from the simulation results that the PMF algorithm has worst performance than all other MSE algorithms. The proposed SUB-COV algorithm performance is similar to the JMMSE-COV and SUB algorithms. At high $S N R_{1}$, the proposed OPT-COV algorithm shows better 
BER performance than the NAF, PMF, SUB, JMMSE-COV and SUB-COV algorithms.

\section{CONCLUSION}

We derived the optimal structure of the AF MIMO relay precoder matrix to minimize the MSE of the symbol estimation at the destination in the presence of the direct source-destination link. We assumed that the relay knows the full CSI of the source-relay link and the partial CSI (covariance feedback) of the direct source-destination link and the relay-destination link. Simulation results demonstrate that the proposed iterative based optimal covariance algorithm has better performance in terms of BER as compared to the conventional covariance feedback based MSE algorithms.

\section{APPENDIX}

Regarding the convexity of (29) for $\widetilde{\mathbf{H}}_{\omega_{0}}^{H} \widetilde{\mathbf{H}}_{\omega_{0}}$ and $\widetilde{\mathbf{H}}_{\omega_{2}}^{H} \widetilde{\mathbf{H}}_{\omega_{2}}$, please note that by using the matrix inversion lemma (17), the MSE function (29) can be rewritten as

$$
\begin{aligned}
E_{\mathbf{H}_{\omega_{0}, 2}}[J(\widetilde{\mathbf{G}})] & =\sigma_{x}^{2} \sigma_{1}^{2} E_{\mathbf{H}_{\omega_{0,2}}}\left[\operatorname { t r } \left\{\left[\sigma_{1}^{2} \mathbf{I}_{N_{S}}+\sigma_{x}^{2} \sigma_{1}^{2} \mathbf{V}_{\Sigma_{0}} \boldsymbol{\Lambda}_{\Sigma_{0}}^{1 / 2}\right.\right.\right. \\
& \times\left[\widetilde{\mathbf{H}}_{\omega_{0}}^{H} \widetilde{\mathbf{H}}_{\omega_{0}}\right] \boldsymbol{\Lambda}_{\Sigma_{0}}^{1 / 2} \mathbf{V}_{\Sigma_{0}}^{H}+\sigma_{x}^{2} \mathbf{V}_{1} \boldsymbol{\Lambda}_{1}^{1 / 2} \widetilde{\mathbf{G}}^{H} \boldsymbol{\Lambda}_{\Sigma_{2}}^{1 / 2} \\
& \times\left(\boldsymbol{\Lambda}_{\Sigma_{2}}^{1 / 2} \widetilde{\mathbf{G}} \widetilde{\mathbf{G}}^{H} \boldsymbol{\Lambda}_{\Sigma_{2}}^{1 / 2}+\frac{\sigma_{2}^{2}}{\sigma_{1}^{2}}\left[\widetilde{\mathbf{H}}_{\omega_{2}}^{H} \widetilde{\mathbf{H}}_{\omega_{2}}\right]^{-1}\right)^{-1} \\
& \left.\left.\left.\times \boldsymbol{\Lambda}_{\Sigma_{2}}^{1 / 2} \widetilde{\mathbf{G}} \boldsymbol{\Lambda}_{1}^{1 / 2} \mathbf{V}_{1}^{H}\right]^{-1}\right\}\right] .
\end{aligned}
$$

From [20], $f(\mathbf{X})=\mathbf{X}^{-1}$ is a matrix-convex function of $\mathbf{X}$. Hence, the MSE function (29) is a convex function for $\widetilde{\mathbf{H}}_{\omega_{0}}^{H} \widetilde{\mathbf{H}}_{\omega_{0}}$ and $\widetilde{\mathbf{H}}_{\omega_{2}}^{H} \widetilde{\mathbf{H}}_{\omega_{2}}$.

\section{REFERENCES}

[1] J. N. Laneman, D. N. C. Tse, and G. W. Wornell, "Cooperative diversity in wireless networks: Efficient protocols and outage behavior," IEEE Trans. Inform. Theory, vol. 50, pp. 3062 - 3080, Dec. 2004.

[2] A. Wittneben and B. Rankov, "Impact of cooperative relays on the capacity of rank-deficient MIMO channels," in Proc. 12th IST Summit on Mobile and Wireless Communications, Aveiro, Portugal, pp. 421-425, June 2003.
[3] J. N. Laneman and G. W. Wornell, "Distributed space-time-coded protocols for exploiting cooperative diversity in wireless networks," IEEE Trans. Inform. Theory, vol. 49, pp. 2415-2425, Oct. 2003.

[4] O. Muñoz-Medina, J. Vidal, and A. Agustín, "Linear transceiver design in non-regenerative relays with channel state information," IEEE Trans. Signal Process., vol. 55, pp. 2593 - 2604, June 2007.

[5] X. Tang and Y. Hua, "Optimal design of non-regenerative MIMO wireless relays," IEEE Trans. Wireless Commun., vol. 6, pp. 1398 - 1407, Apr. 2007.

[6] W. Guan and H. Luo, "Joint MMSE transceiver design in non-regenerative MIMO relay systems," IEEE Commun. Lett., vol. 12, pp. 517 - 519, July 2008.

[7] A. S. Behbahani, R. Merched, and A. M. Eltawil, "Optimizations of a MIMO relay network," IEEE Trans. Signal Process., vol. 56, pp. 5062 5073, Oct. 2008.

[8] Y. Rong, X. Tang, and Y. Hua, "A unified framework for optimizing linear non-regenerative multicarrier MIMO relay communication systems," IEEE Trans. Signal Process., vol. 57, pp. 4837-4851, Dec. 2009.

[9] Y. Rong and F. Gao, "Optimal beamforming for non-regenerative MIMO relays with direct link," IEEE Commun. Lett., vol. 13, pp. 926-928, Dec. 2009.

[10] Y. Rong, "Optimal joint source and relay beamforming for MIMO relays with direct link," IEEE Commun. Lett., vol. 14, pp. 390-392, May 2010.

[11] F. S. Tseng and W. R. Wu, "Linear MMSE transceiver design in amplifyand-and-forward MIMO relay systems," IEEE Trans. Veh. Technol., vol. 59, no. 2, pp. 754-765, Feb. 2010

[12] H. W. Je, B. Lee, S. Kim, and K. B. Lee, "Design of non-regenerative MIMO-relay systems with partial channel state information," in Proc. IEEE Int. Conf. Commun, Beijing, 2008, pp. 4441-4445.

[13] C. Jeong and H.-M. Kim, "Precoder design of non-regenerative relays with covariance feedback," IEEE Commun. Lett., vol. 13, no.12, pp.920 - 922, Dec. 2009.

[14] K. Dae-Hyun and H.-M. Kim, "MMSE precoder design for a nonregenerative MIMO relay with covariance feedback," in Proc. IEEE 21st Int. Sym. Per. Ind. Mobile Radio Commun., Istanbul, Turkey, pp. 461-464, Aug. 2010.

[15] L. Gopal, Y. Rong, and Z. Zang, "Joint MMSE transceiver design in nonregenerative MIMO relay systems with covariance feedback", in Proc. IEEE 17th Asia-Pacific Conf. Commun., Sabah, Malaysia, Oct. 2011.

[16] D. Shiu, G. J. Foschini, M. J. Gans, and J. M. Kahn, "Fading correlation and its effect on the capacity of multielement antenna systems," IEEE Trans. Commun., vol. 48, no. 3, pp. 502-513, Mar. 2000.

[17] S. M. Kay, "Fundamentals of Statistical Signal Processing: Estimation Theory," Englewood Cilffs, NJ: Prentice Hall, 1993.

[18] A. W. Marshall, I. Olkin and B. C. Arnold, "Inequalities: Theory of majorization and its applications," New York, Springer, 2011.

[19] E. K. P. Chong and S. H. Zak, "Introduction to Optimization," New York, NY:Wiley-Interscience, 2001.

[20] T. Ando, "Concavity of certain maps on positive definite matrices and applications to Hadamard products," Linear Algebra and Its Applications, vol. 26, pp. 203-241, Aug. 1979. 